\title{
Resilience and vulnerability of maternity services in Zimbabwe: a comparative analysis of the effect of Covid-19 and lockdown control measures on maternal and perinatal outcomes at Mpilo Central Hospital.
}

Clare Shakespeare ( $\nabla$ clare.shakespeare@gmail.com )

Mpilo Central Hospital https://orcid.org/0000-0002-9084-0673

H Dube

Mpilo Central Hospital

S Moyo

Mpilo Central Hospital

S Ngwenya

Mpilo Central Hospital

Research article

Keywords: Covid-19, indirect maternal outcomes, indirect perinatal outcomes, vulnerability, resilience, healthcare systems

Posted Date: August 12th, 2020

DOl: https://doi.org/10.21203/rs.3.rs-52159/v1

License: (c) (1) This work is licensed under a Creative Commons Attribution 4.0 International License. Read Full License 


\section{Abstract}

Background: On $27^{\text {th }}$ March the Zimbabwean government declared the Covid-19 pandemic a 'national disaster'. Travel restrictions and emergency regulations have had significant impacts on maternity services, including staff shortages, resource stock-outs, and closure of antenatal clinics. Estimates of the indirect impact of Covid-19 on maternal and perinatal mortality expect it to be considerable, but little data is yet available.

This study aims to examine the impact of Covid-19 and lockdown control measures on non-Covid outcomes in a government tertiary level maternity unit in Bulawayo, Zimbabwe, by comparing maternal and perinatal morbidity and mortality before and after lockdown was implemented.

Methods: This is a retrospective, observational study, using a cross-sectional design to compare routine monthly maternal and perinatal statistics three months before and after Covid-19 emergency measures at Mpilo Central Hospital.

Results: Between January-March and April-June 2020, average monthly deliveries fell from 747 to 681 and Caesarean section rates from $29.8 \%$ to $26.6 \%$. Women with unbooked pregnancies presenting in labour almost doubled from $4.4 \%$ to $8 \%$. There was no substantial change, however, in maternal mortality or severe maternal morbidity (post-partum haemorrhage (PPH), uterine rupture, severe preeclampsia/eclampsia), stillbirth rate or special care baby unit (SCBU) admission. There was a small increase in early neonatal death (ENND) from an average of 18.7 to 24.0 deaths per month.

Conclusion: Maternal and perinatal outcomes must continue to be monitored to assess the impact of Covid-19 and lockdown measures as the epidemic in Zimbabwe unfolds. Despite the vulnerability of the healthcare system, the resilience and commitment of maternity units and healthcare workers to providing care in the most difficult circumstances should not be underestimated.

\section{Introduction}

In March 2020, Covid-19 was recognised by the WHO as a global pandemic. Although Africa appears so far to have been comparatively spared(1), limited health system capacity for testing and disease surveillance restricts assessment of the true size and impact of the epidemic in many African countries(2). Widespread use of non-pharmacological measures to control the epidemic include international, regional and local travel restrictions, school and workplace closures, and limitations on social gatherings. Whilst likely cost-effective in high income countries(3), the feasibility and effectiveness of such lockdown interventions has been questioned in the context of sub-Saharan Africa with younger population age structures, predominance of informal economies and weak existing healthcare systems $(4$, $5)$.

Despite low numbers of cases, and limited direct morbidity and mortality related to Covid-19, health care systems in many southern African countries remain vulnerable to indirect effects on non-Covid-19 health 
outcomes, especially maternal and newborn health(6). Fear of the virus, shortages of resources, and disruption of healthcare infrastructure and systems as a result of lockdown measures, present increased barriers for access to healthcare services and may result in breakdown of routine health programmes $(6$, 7). Similar issues during the Ebola outbreak in west Africa in 2014 resulted in a sustained reduction in use of essential maternal and child health services(8). In some cases, indirect effects on health were more extensive than the epidemic itself(9). Already in some European countries reduction in use of child health services has been observed during the Covid-19 pandemic, raising fears about additional morbidity and mortality resulting from delayed presentations $(10,11)$. Models based on estimates of disruption to routine maternal and child health services and food insecurity as an indirect result of Covid-19 in low and middle income countries (LMICs) predict up to $38.6 \%$ increase in maternal deaths per month across 118 countries(6).

Zimbabwe has had a limited epidemic so far, with 1478 cases and 25 deaths as of 19th July 2020(12). Fewer than ten cases have been officially reported in pregnant women (Mpilo Rapid Response Team, personal communication, 20th July 2020). The Zimbabwean government declared Covid-19 a national disaster on 27th March. A nationwide lockdown commenced on 1st April. All non-essential travel, shops and services were suspended for 5 weeks, with gradual relaxation of measures since to re-open shops and businesses. Although healthcare was always exempted from lockdown measures, ongoing issues for healthcare services include transport difficulties for patients and staff, and financial hardship resulting from loss of livelihoods. These difficulties present additional barriers for affordability and access to healthcare.

Mpilo Central Hospital is a government tertiary referral hospital in Bulawayo. The maternity department receives high risk cases and referrals from the whole of southern Zimbabwe (Matabeleland North, Matabeleland South and Midland provinces). Many essential services, including antenatal clinics, have closed and all elective work, including elective Caesarean sections, has been cancelled since April 2020 due to the Covd-19 pandemic. Ongoing shortages of medical and midwifery staff have been exacerbated with up to $20 \%$ of midwifery staff off-duty due to isolation or quarantine measures during May 2020 .

An increasing number of individual studies and systematic reviews are providing evidence on the impact of Covid-19 on pregnancy outcomes $(13,14)$. Little is available on the indirect impacts on maternal and perinatal morbidity and mortality, especially in sub-Saharan Africa. This study aimed to examine the impact of Covid-19 and lockdown control measures on non-Covid outcomes at Mpilo Central Hospital in Bulawayo, Zimbabwe. The objectives were to compare maternal and perinatal outcomes before and after lockdown was implemented. The hypothesis was that both morbidity and mortality would have increased.

\section{Materials And Methods}

This is a retrospective, observational study, using a cross-sectional design to compare routine monthly maternal and perinatal statistics before and after the implementation of Covid-19 lockdown measures at 
Mpilo Central Hospital in Bulawayo, Zimbabwe.

Maternal and perinatal statistics are routinely collected and collated by reproductive health officers every month from hospital records and daily reports. These routine data were used to compare outcomes for three months before and after lockdown (January-March vs April-June 2020). These months were chosen as a comparator to keep other factors as similar as possible as Zimbabwe has experienced many other disruptions to services which may affect outcomes, such as industrial action by medical staff during 2019.

Data was chosen to reflect four aspects of maternity care: workload, antenatal care, maternal morbidity and mortality, perinatal morbidity and mortality (Table 1). According to Donabedian's framework for assessment of quality of care(15), the selected indicators of workload and antenatal care represent process measures, while maternal and perinatal morbidity and mortality indicators represent outcome measures.

There is no global consensus on which maternal and perinatal indicators should be chosen to represent quality and outcomes of maternity care(16), these indicators were chosen to reflect and correspond with a number of reviews on the topic(16-18), as well as to fit data that is routinely recorded and available at Mpilo.

Ethics approval was obtained from the Mpilo Research Ethics Committee.

\section{Results}

Overall results are presented in Table 2.

\section{Workload:}

The average number of deliveries conducted each month reduced from 747 in the first quarter of 2020 to 681 after lockdown (Fig. 1). The number of Caesarean sections also reduced, both as absolute number performed (average 222 per month versus 181), and as Caesarean section rate (29.8\% versus $26.6 \%$ ).

\section{Antenatal care:}

In the second quarter of 2020, the percentage of women delivering at Mpilo who were booked at the hospital fell from $41.6-35.9 \%$. The number of women presenting in labour with unbooked pregnancies almost doubled from $4.4-8 \%$. There was a small increase in the number of women referred in from other health centres $(54.6 \%$ vs $56.3 \%)$.

\section{Maternal morbidity and mortality:}


5 maternal deaths were recorded in January-March 2020, and 3 maternal deaths were recorded in AprilJune 2020. There was no considerable change in rates of PPH, or severe pre-eclampsia/eclampsia. There were more cases of uterine rupture reported in the second quarter of 2020 , but the numbers remain very small.

\section{Perinatal morbidity and mortality:}

Stillbirth rate fell slightly from an average of 33.1/1000 deliveries in January-March, to 30.9/1000 deliveries in April-June. The average total number of early neonatal deaths (ENND) increased (18.7 versus 24.0) while the percentage of admissions to Special Care Baby Unit (SCBU) remained stable (23.95 versus $22.7 \%$ ).

\section{Discussion}

A major strength of this study is that it relies on routinely collected and contemporaneously reported data from the maternity department. This means that data is readily and quickly available to monitor outcomes and trends, and any systematic errors in data collection are likely to remain constant from month to month. However, it is possible that if wards are short-staffed and staff working under additional pressure, this may affect the way they report outcomes, for example, being less likely to recognise and report cases of post-partum haemorrhage (PPH).

Another limitation is that the measures chosen may not perfectly represent the outcomes of interest. For example, antenatal care booking status represents coverage, but not quality of antenatal care provided. Additionally, none of the indicators selected represent important structural measures of quality, such as staff shortages and drug stock-outs, both of which have occurred before and during the Covid-19 lockdown period. Finally, our findings must be interpreted in the context of the limited epidemic and time period (three months) of Covid-19 and control measures in Zimbabwe thus far. Outcomes may continue to change and it will be important to monitor this.

Workload: The overall reduction in number of deliveries being conducted at Mpilo raises the question of where women are delivering instead. It would be interesting to examine statistics from other clinics within the city to see if their deliveries have increased. More women may choose to deliver locally, perceiving increased risk of Covid-19 at the central hospital or difficulties with transport. Some families moved back from urban to rural areas when lock-down was announced and may be delivering at rural clinics and district general hospitals. The critical question is whether these women are still delivering in safe environments and complications recognised.

Fewer Caesarean sections are being done, this may be in part due to cancellation of elective Caesarean sections, but also reduced capacity in maternity theatres associated with resource and staffing shortages (due to illness or quarantine). Emergency Caesarean sections are known to be associated with more 
complications for mother and baby than elective procedures, however these outcomes are not captured by our data.

Antenatal care: The number of women presenting in labour with an unbooked pregnancy has almost doubled since Covid-19 lockdown measures were instituted. The antenatal clinic at Mpilo has been closed since April 2020, and no women have been able to book their pregnancies there. This trend is therefore likely to continue until antenatal services are restored. Women may be booking elsewhere, for example at local clinics, which in time may be reflected in an increase in referrals from other health centres. We would expect an increase in adverse outcomes to occur as fewer women access antenatal care. It may still be too soon, however, to observe the impact on women who would have booked their pregnancies during lockdown and will present for delivery later in the year.

Maternal and perinatal outcomes: Overall there has been no considerable change in maternal outcomes. The small increase in cases of uterine rupture may reflect barriers in accessing and delivering obstetric care on labour ward, however the numbers for all the outcomes are so small that it is difficult to draw any significant conclusions. The increase in early neonatal deaths could be due to problems within the obstetric or neonatal departments at Mpilo, at referring health centres, or delayed presentation associated issues within the community such as transport. Further investigation would be needed to clarify these issues.

Finally, the data on maternal deaths recorded here does not capture deaths occurring outside hospital or at other health centres and hospitals within the region. It would be important to explore this data for the whole region when it is available, to see whether women are experiencing excess morbidity and mortality outside Mpilo, unable to access the central hospital itself.

Similarly to many southern African countries, the healthcare system in Zimbabwe is fragile and vulnerable to the effects of internal and external crises such as Covid-19(19). In Zimbabwe, the Covid-19 pandemic has occurred on the background of a weakened healthcare system that has experienced persistent shortages of resources and underfunding. A prolonged junior doctors strike in 2019 lasted four months from October, ending at the beginning of January 2020.

We were surprised by our findings, that did not fit our hypothesis that maternal and perinatal morbidity and mortality would increase during the lockdown period. Our experience of working during this time has been of increased challenges and adversity associated with logistical, resource, and staffing difficulties. Interestingly, a study of the effects of Covid-19 lockdown measures on access to primary care in rural South Africa had similar unexpected results(20).

Explanations for our results include statistics being recorded differently during the lock-down period due to other pressures on staff time, adverse outcomes not being captured as they occur outside of the hospital environment, or that it is still too soon to see the result of impacts such as lack of antenatal care. Women delivering now are likely to still have had some, if limited, antenatal care. 
Even so, the results presented here could also be seen to represent the significant efforts of healthcare workers and maternity services showing remarkable resilience in difficult circumstances, with great commitment to keeping women and their babies safe. Several elements of organisational design, commonly used to analyse strategic success(21), may describe the way that resilience is manifested at Mpilo hospital to prevent adverse outcomes. The structure and systems of the Mpilo maternity department are oriented to identifying women at risk of complications and intervening early. The focus is on reducing morbidity and preventing mortality by early recognition and timely involvement of senior midwifery and medical staff. Regular rounds by maternity matrons identify issues and escalate promptly to consultants if necessary. Both medical and midwifery staff are resilient and adaptable, with experience of working in difficult conditions. They are skilled at responding to complex emergencies and scenarios on labour ward such as eclampsia, uterine rupture and major PPH, with competencies such as Caesarean hysterectomy as routine. They are conditioned to working with suboptimal availability of resources and improvising. There is a shared value of commitment to keeping women and babies safe despite a lack of resources. Finally, leadership style in the department tends to be formal and directive, with an emphasis on accountability. This may not suit all environments but can provide clear and consistent guidance when trying to achieve specific goals and maintain standards in challenging circumstances such as those presented by Covid-19.

While the results presented here are positive, it will be essential to continue monitoring outcomes as the epidemic in Zimbabwe escalates and further lockdown measures and changes to services are being made. There is still potential for poor maternal and perinatal outcomes if access to healthcare remains restricted and healthcare workers are increasingly burnt out by chronic under-staffing and difficult working conditions.

\section{Conclusion}

Healthcare systems in southern Africa are vulnerable to the indirect effects of Covid-19. It is important that we continue to carefully monitor maternal and perinatal outcomes to assess the impact of lockdown measures on women and babies as the epidemic in Zimbabwe unfolds. The resilience and commitment of maternity units and healthcare workers to providing care to these groups in the most difficult of circumstances, however, should not be underestimated.

\section{Declarations}

\section{Ethics}

Ethical approval was obtained from the Mpilo Research Ethics Committee

\section{Consent for publications:}

$\mathrm{N} / \mathrm{A}$ 


\section{Availability of data and materials:}

The datasets used during the current study are available from the corresponding author on reasonable request.

\section{Competing interests:}

The authors declare that they have no competing interests.

\section{Funding:}

No source of funding was required for this study.

\section{Author contributions:}

CS and HD designed the study and collected the data. All authors contributed to analysis of data and interpretation of findings. CS was responsible for writing the first draft, and all authors contributed to finalising the manuscript.

\section{Acknowledgements:}

Special thanks to Sr S Mhlanga and Sr N Moyo from the Reproductive Health Office for their assistance with providing the maternity department data.

\section{References}

1. WHO

WHO. Coronavirus disease. (Covid-2019) situation reports [Internet]. 2020 [accessed 2020 Jun 24].

Available from: https://www.who.int/emergencies/diseases/novel-coronavirus-2019/situationreports.

2. Paintsil E. COVID-19 threatens health systems in sub-Saharan Africa: the eye of the crocodile. J Clin Invest [Internet]. 2020 Apr 27;130(6):2741-4. Available from: https://www.jci.org/articles/view/138493.

3. Barnett-Howell Z, Mobarak A. Should Low-Income Countries Impose the Same Social Distancing Guidelines as Europe and North America to Halt the Spread of COVID-19? Yale Sch Manag. 2020.

4. Dahab M, Zandvoort K, Flasche S, Warsame A, Spiegel P, Waldman R, et al. COVID-19 control in lowincome settings and displaced populations: what can realistically be done? [Internet]. LSHTM news. 
[accessed 2020 Jun 24]. Available from: https://www.Ishtm.ac.uk/newsevents/news/2020/covid-19control-low-income-settings-and-displaced-populations-what-can\#.XnpKCXF93Fc.email.

5. Mehtar S, Preiser W, Lakhe NA, Bousso A, TamFum J-JM, Kallay O, et al. Limiting the spread of COVID-19 in Africa: one size mitigation strategies do not fit all countries. Lancet Glob Heal [Internet]. $2020 \mathrm{Jul} ; 8(7): \mathrm{e} 881-3$. Available from:

https://linkinghub.elsevier.com/retrieve/pii/S2214109X20302126.

6. Roberton T, Carter ED, Chou VB, Stegmuller AR, Jackson BD, Tam Y, et al. Early estimates of the indirect effects of the COVID-19 pandemic on maternal and child mortality in low-income and middleincome countries: a modelling study. Lancet Glob Heal [Internet]. 2020 Jul;8(7):e901-8. Available from: https://linkinghub.elsevier.com/retrieve/pii/S2214109X20302291.

7. $10.1186 / \mathrm{s} 41182-020-00219-6$

Amimo F, Lambert B, Magit A. What does the COVID-19 pandemic mean for HIV, tuberculosis, and malaria control? Trop Med Health [Internet]. 2020 Dec 13;48(1):32. Available from: https://tropmedhealth.biomedcentral.com/articles/10.1186/s41182-020-00219-6.

8. Delamou A, Ayadi AM, El, Sidibe S, Delvaux T, Camara BS, Sandouno SD, et al. Effect of Ebola virus disease on maternal and child health services in Guinea: a retrospective observational cohort study. Lancet Glob Heal [Internet]. 2017 Apr;5(4):e448-57. Available from: https://linkinghub.elsevier.com/retrieve/pii/S2214109X17300785.

9. Elston JWT, Cartwright C, Ndumbi P, Wright J. The health impact of the 2014-15 Ebola outbreak. Public Health [Internet]. 2017 Feb;143:60-70. Available from: https://linkinghub.elsevier.com/retrieve/pii/S0033350616303225.

10. Royal College of Paediatrics and Child Health. COVID-19 - statement on delayed presentations [Internet]. [cited 2020 Jun 26]. Available from: https://www.rcpch.ac.uk/news-events/news/covid-19statement-delayed-presentations.

11. Lazzerini M, Barbi E, Apicella A, Marchetti F, Cardinale F, Trobia G. Delayed access or provision of care in Italy resulting from fear of COVID-19. Lancet Child Adolesc Heal [Internet]. 2020 May;4(5):e10-1. Available from: https://linkinghub.elsevier.com/retrieve/pii/S2352464220301085.

12. WHO. WHO Covid-19: Zimbabwe situation [Internet]. 2020 [cited 2020 Jul 20]. Available from: https://covid19.who.int/region/afro/country/zw.

13. $10.1002 /$ uog. 22088

Juan J, Gil MM, Rong Z, Zhang Y, Yang H, Poon LC. Effects of coronavirus disease 2019 (COVID-19) on maternal, perinatal and neonatal outcomes: a systematic review. Ultrasound Obstet Gynecol [Internet]. 2020 May 19;uog.22088. Available from:

https://onlinelibrary.wiley.com/doi/abs/10.1002/uog.22088.

14. $10.1002 /$ ijgo.13182

Elshafeey F, Magdi R, Hindi N, Elshebiny M, Farrag N, Mahdy S, et al. A systematic scoping review of COVID-19 during pregnancy and childbirth. Int J Gynecol Obstet [Internet]. 2020 Jul 17;150(1):47-52. Available from: https://onlinelibrary.wiley.com/doi/abs/10.1002/ijgo.13182. 
15. Donabedian A. Evaluating the Quality of Medical Care. Millbank Q. 2005;83(4):691-729.

16. 10.1186/s12884-019-2173-2

Saturno-Hernández PJ, Martínez-Nicolás I, Moreno-Zegbe E, Fernández-Elorriaga M, PoblanoVerástegui $\mathrm{O}$. Indicators for monitoring maternal and neonatal quality care: a systematic review. BMC Pregnancy Childbirth [Internet]. 2019 Dec 11;19(1):25. Available from: https://bmcpregnancychildbirth.biomedcentral.com/articles/10.1186/s12884-019-2173-2.

17. Sibanda T, Fox R, Draycott TJ, Mahmood T, Richmond D, Simms RA. Intrapartum care quality indicators: a systematic approach for achieving consensus. Eur J Obstet Gynecol Reprod Biol [Internet]. 2013 Jan;166(1):23-9. Available from: https://linkinghub.elsevier.com/retrieve/pii/S0301211512004411.

18. https://dx.plos.10.1371/journal.pone.0211955 Sauvegrain P, Chantry AA, Chiesa-Dubruille C, Keita H, Goffınet F, Deneux-Tharaux C. Monitoring quality of obstetric care from hospital discharge databases: A Delphi survey to propose a new set of indicators based on maternal health outcomes. Ford JB, editor. PLoS One [Internet]. 2019 Feb 12;14(2):e0211955. Available from: https://dx.plos.org/10.1371/journal.pone.0211955.

19. Menendez C, Gonzalez R, Donnay F, Leke RGF. Avoiding indirect effects of COVID-19 on maternal and child health. Lancet Glob Heal [Internet]. 2020 Jul;8(7):e863-4. Available from: https://linkinghub.elsevier.com/retrieve/pii/S2214109X20302394.

20. Siedner MJ, Kraemer JD, Meyer MJ, Harling G, Mngomezulu T, Gabela P, et al. Access to primary healthcare during lockdown measures for COVID-19 in rural South Africa: a longitudinal cohort study. medRxiv Prepr Serv Heal Sci. 2020;2020.05.15.20103226.

21. Goodwin N, Gruen R, lles V. Chapter 17 Making change happen. In: Managing health services. Open University Press; 2006. p. 184-99.

\section{Tables}

Due to technical limitations, the tables are provided in the Supplementary Files section.

\section{Figures}




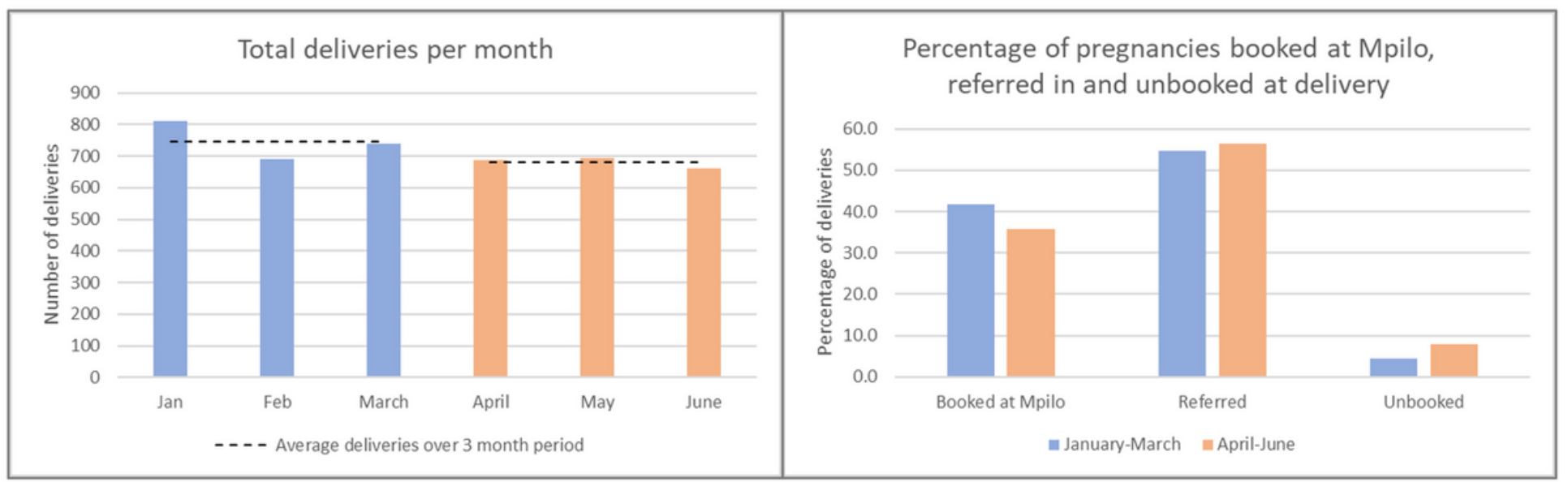

Figure 1

Trends in total deliveries and percentage of Mpilo booked pregnancies, referrals, and unbooked pregnancies at delivery.

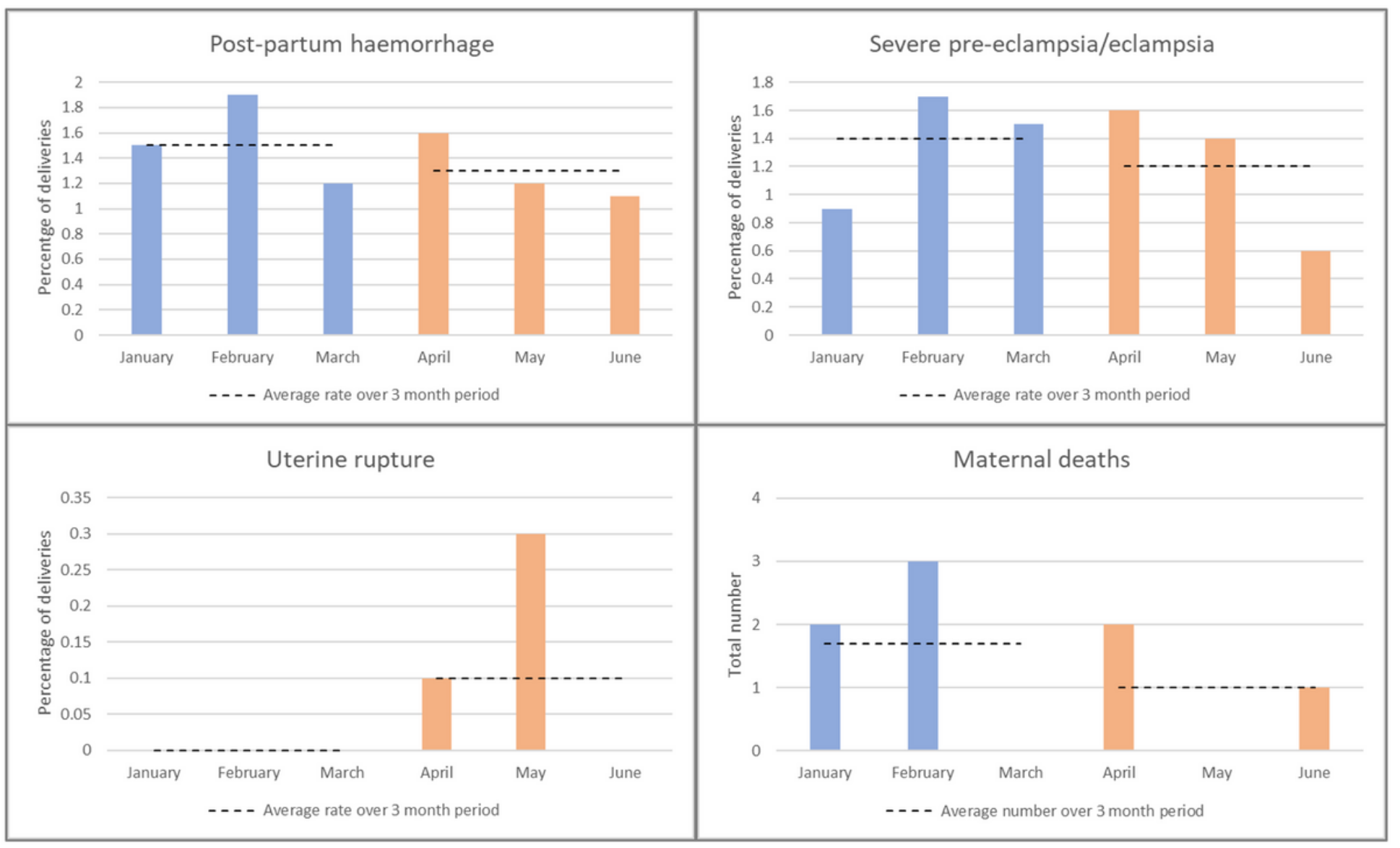

\section{Figure 2}

Maternal morbidity and mortality 


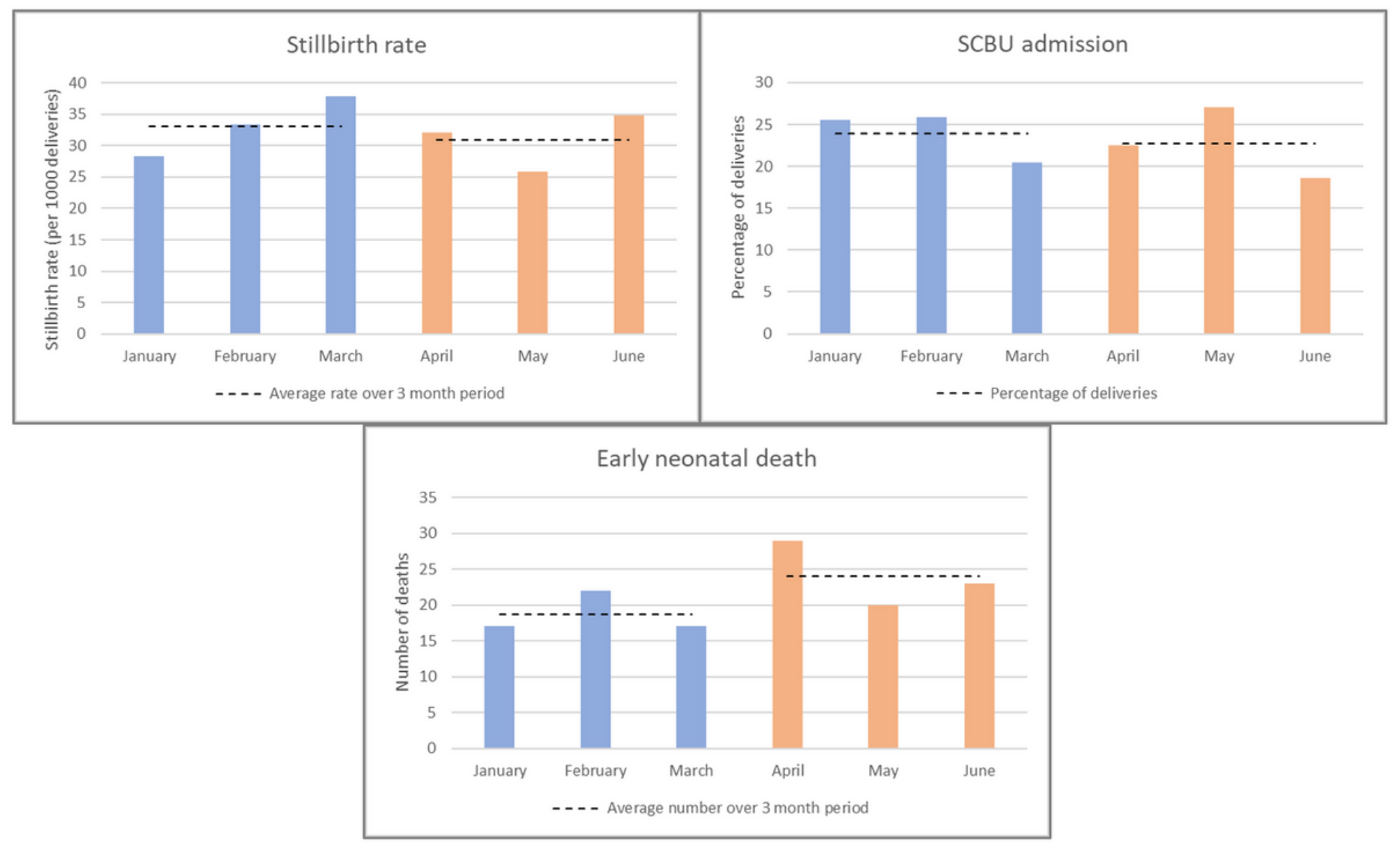

Figure 3

Perinatal morbidity and mortality

\section{Supplementary Files}

This is a list of supplementary files associated with this preprint. Click to download.

- Tables.docx 